\title{
Cancer Death Risk Related to Radiation Exposure from Computed Tomography Scanning Among Testicular Cancer Patients
}

\author{
EEVA SALMINEN $^{1,2}$, HANNELE NIINIVIITA $^{3,4}$, HANNU JÄRVINEN $^{1}$ and SIRPA HEINÄVAARA ${ }^{5}$ \\ ${ }^{1}$ STUK-Radiation and Nuclear Safety Authority, Helsinki, Finland; \\ ${ }^{2}$ Department of Oncology, Turku University Hospital, Turku, Finland; \\ ${ }^{3}$ Department of Medical Physics, Turku University Hospital and University of Turku, Turku, Finland; \\ ${ }^{4}$ Medical Imaging Center of Southwest Finland, Turku University Hospital, Turku, Finland; \\ ${ }^{5}$ Finnish Cancer Registry, Helsinki, Finland
}

\begin{abstract}
Background: A study of the computed tomography (CT) imaging related effective doses and radiation-related cancer death risk. Patients and Methods: Estimate effective doses were computed from CT scans of testicular cancer patients treated and followed-up in Turku University Hospital, South Western Finland. Association between effective doses from follow-up CT scans and radiation-induced cancer death was examined using United Nations Scientific Committee on the Effects of Atomic Radiation (UNSCEAR) 2008 formula. Results: Mean effective dose per CT abdomen was 9.32 (standard deviation, SD 3.89) $\mathrm{mSv}$ and for whole-body CT it was 14.24 (SD 6.84) $m S v$. During follow-up of 6 years, the patients were estimated to undergo 12 to 14 abdominal/whole-body CTs and the corresponding risk estimates were 0.11 and 1.14, respectively. The risk of estimated radiation-induced cancer deaths (RICD in \%) computed for mean effective doses was lower in patients diagnosed at older age, being 0.61 for 10 19 years age and 0.04 for 40-49 years age at the diagnosis. Conclusion: Patient radiation exposure in CT imaging is associated with the type of CT device and imaging protocols, which should be periodically updated and reviewed to minimize individual exposure. Using the UNSCEAR modelling $2 \%$ risk for radiation related cancer death was attributed to diagnostic exposure of study patients. Age at the diagnosis was associated with $C T$ imaging related radiation exposure. The highest exposure was estimated to the youngest patients.
\end{abstract}

Correspondence to: Eeva K. Salminen, MD, P.O. Box 52, Savitehtaankatu 1, FI-20521 Turku, Finland. Tel: +35 89559881, Fax: +358975988500,e-mail: eevsal@utu.fi

Key Words: Computed tomography, radiation exposure, cancer death risk.
Computed tomography (CT) in diagnostic imaging exposes patients for a substantial part of their lifetime exposure to cumulative medical radiation dose. With regard to the oncological population, multiple follow-up CTs are being justified by the need to promptly evaluate treatment effects or to localize cancer spread or recurrence (1). Nevertheless, with frequent CT imaging there is a concern for patients being exposed to high effective doses. Among cancer patients, particularly adolescence and young adult (AYAs) cancer patients, with excellent cure prospects are of concern. Testicular cancer patients fall into the AYA group.

In testicular cancer the recommended imaging frequency is high and it depends on the primary treatment or follow-up policy. It usually includes several whole-body or abdominal scans annually for the first 2-3 years of follow-up and twice a year for another 2-3 years and even annually thereafter. In five years' time patients may undergo 12 to 14 abdominal or whole-body CT imaging (2).

The effective dose, according to the International Commission on Radiological Protection ICRP report is a descriptor that can be used to characterize radiation exposure of patients to CT $(3,4)$. We have recorded data and calculated effective and organ doses from CT scans of testicular cancer patients (5). The aim of this report was to study the association of effective dose from multiple abdominal and whole-body CTs to obtain a cancer death risk related to radiation exposure from CTs according to current follow-up CT frequency in testicular cancer patient population.

\section{Patients and Methods}

Patients. A cohort of 115 testicular cancer patients scanned between 1995-2011 was studied to define the average effective dose (mean, $\mathrm{SD})$ from abdominal and whole body CTs done for the diagnosis and/or follow up of testicular cancer using different scanners in the observation period and summing them over all patients and age groups. 
Table I. Ranges of effective doses (mSv) per abdominal or whole body CT imaging with (c.m.) or without (nat) i.v. contrast for different scanners.

\begin{tabular}{lcccccc}
\hline & Abdomen nat. & Abdomen c.m.-ct & Abdomen nat+c.m.-ct & Body nat. & Body c.m.-ct & Body nat+c.m.-ct \\
\hline Siemens Somatom Plus 4 & $3.6-4.1$ & $3.7-6.5$ & $5.6-8$ & $5.3-9.3$ & $5.1-9.2$ & $6-15.4$ \\
Siemens Volume Zoom & $6.2-9.8$ & 8.4 & $12.6-19.3$ & - & $12-13.9$ & $15.4-27$ \\
Toshiba Asteion dual & - & 12 & $15.8-17.6$ & $14-17$ & $15.5-21$ & $21.1-25.9$ \\
GE Pace & $11-18$ & $11-16$ & 22.8 & $15-21$ & $16-22$ & $26.1-27.3$ \\
GE LightSpeed 16 & $6.6-17$ & $4.9-14.7$ & $14.3-20.6$ & $10.3-26$ & $8.1-17.1$ & $11.3-24.4$ \\
Picker International inc. PQ-2000 & - & $6-14.5$ & $8-12.9$ & - & $12.9-15$ & $17.8-20.2$ \\
\hline
\end{tabular}

C.m, Contrast medium; i.v., intravenous; ct, computed tomography.

The patients aged 16 to 48 years underwent 522 CTs while being treated and followed up at the Department of Radiotherapy and Oncology, Turku University Hospital. Mean age (and SD) at the first CT scan was 29 (7.1) years. Details of radiological investigations were obtained from the institutional radiology comprised database.

All the studies for the cohort had been done with fifteen different scanners representing the available scanners in Finland during the observation period. The most frequently used scanners were Somatom Plus 4 and Volume Zoom (Siemens, Erlangen, Germany), Pace and LightSpeed 16 (GE, Milwaukee, WI, USA), Asteion dual (Toshiba, Otawara, Japan) and International PQ-2000 (Picker, New York city, NY, USA) since 2001. From all of the studies $94 \%$ were made with these scanners.

Table I presents the highest and the lowest effective doses when using the above mentioned scanners. When contrast medium was used the whole abdominal region was examined. Alternatively, the organ of interest was at first examined without the contrast medium and after that the whole region with contrast medium.

Other CT examinations were done on head, thorax, urinary organs, respiratory ducts, lumbar spine and blood vessels. In addition, 21 CT scans were carried out for radiotherapy dose planning. The effective dose of the examinations was between 5.9 $\mathrm{mSv}$ and $14 \mathrm{mSv}$ depending on the scanner and the length of the scan. These were not included in this data.

Since the exact diagnosis and follow-up data were not complete for all patients, hypothetical diagnoses and follow-ups with CT scans were assumed. All patients were assumed to be either nonseminoma patients and to be followed for six years with the standard treatment protocol or seminoma patients and to be followed for eight years.

Statistical analyses. This study is descriptive, and therefore estimates of RICD have not been compared between seminoma and non-seminoma patients. An average and range of effective doses were calculated for each patient. Mean and median describe the average effective dose, and minimum and maximum describe the smallest and the largest effective doses had all scans been done at the lowest or the highest doses. Estimates of radiation related cancer death (RICD in \%) were then calculated by multiplying patient's each effective dose estimates (mean, median, minimum, maximum) at a given age with age-specific UNSCEAR coefficient (6=UNSCEAR 2008) and summing them over all patients by age group at scan.

\section{Results}

Highest cumulative effective doses were from patients who had undergone many examinations or whose examinations were performed with an older scanner. The patient who had 26 examinations had the highest cumulative effective dose of $282.1 \mathrm{mSv}$. Seventy-six $(66 \%)$ of patients in this study were exposed to more than $50 \mathrm{mSv}$.

Based on the collected imaging data, the mean effective dose per one abdominal CT was $9.32 \mathrm{mSv}$ (SD 3.89) for 90 scans. Most scans were done as whole body scans with the mean effective dose of $14.24 \mathrm{mSv}$, (SD 6.85) for 432 scans.

Tables II and III present the estimates of radiation-induced cancer deaths (RICD in \%) based on UNSCEAR 2008 (6) model. Estimated risk of radiation-induced cancer deaths (RICD in \%) varied according to the age of diagnosis from 0.61 at age between 10-19 years, 0.56 at the age of 20-29, 0.26 at the age of $30-39$, to 0.04 at the age of $40-49$ years.

\section{Discussion}

Adolescent and young adult cancer patients who survive cancer have a higher relative risk of second cancers compared to the general population and other cancer survivors (7). Exposure to CT imaging radiation has been suggested to contribute to this risk $(8,9)$.

As indicated in Table I, even in one hospital where multiple CT devices are used, the dose exposures of the patients can be variable as marked differences were observed related to the type of device and imaging protocols. This is in alignment with previous observation showing significant variation in average effective doses $(\mathrm{mSv})$ for different cancer follow-up protocols among different treatment centers even when comparing single follow-up imaging (1). Vendor recommendations need to be adjusted to the centers protocols.

It has been estimated that even a single CT imaging may associate with increased cancer risk. For subjects exposed to at least one CT scan before the age of 20 years, cancer incidence 
Table II. Radiation-induced cancer death estimates (RICD in \%) for non-seminoma patients in six years follow-up.

\begin{tabular}{lcccc}
\hline Age (years) & Mean & Median & $\min$ & $\max$ \\
\hline $10-19$ & 1.14 & 1.12 & 0.69 & 1.71 \\
$20-29$ & 1.09 & 1.07 & 0.66 & 1.60 \\
$30-39$ & 0.58 & 0.57 & 0.35 & 0.84 \\
$40-49$ & 0.11 & 0.11 & 0.067 & 0.14 \\
\hline
\end{tabular}

Table III. Radiation-induced cancer death estimates (RICD in \%) for seminoma patients in over eight years follow-up.

\begin{tabular}{lcccc}
\hline Age (years) & Mean & Median & $\min$ & $\max$ \\
\hline $10-19$ & 1.31 & 1.28 & 0.79 & 1.97 \\
$20-29$ & 1.26 & 1.24 & 0.77 & 1.86 \\
$30-39$ & 0.70 & 0.69 & 0.43 & 1.02 \\
$40-49$ & 0.16 & 0.15 & 0.10 & 0.21 \\
\hline
\end{tabular}

was increased on average by $24 \%$ (10). Furthermore, such estimates show variability in the increased risk depending on the type of CT examination and the patient's age and sex (11, 12). Radiation doses from identical CT procedures can vary by as much as 10 -fold from facility to facility $(11,13)$.

Testicular cancer patients become exposed to substantial radiation doses form CT examinations during follow-up (9). The risk of second cancers due to $\mathrm{CT}$ related radiation exposure has been suggested to exceed the risk for recurrences in this patient population (1). The overall relative risks of second cancer among testicular cancer survivors range from 1.4- to 2.8-fold (14), albeit the studies looking at the impact of diagnostic radiation exposure have generated inconclusive results. It is unclear how much the radiation exposure of frequent CT scanning, which is recommended for the followup of these patients (15), contributes to the risk increase.

Cancer risk related to imaging radiation exposure has been estimated by Matthews et al. (10). They gave an overall excess risk of about 0.125 cancers per Sievert, which equated to one excess cancer per 1,800 head CTs with an average estimated dose of around $4.5 \mathrm{mSv}$. The effective dose from abdominal and whole body scans can be 2-3 times higher than that. Zagars et al. (16) have estimated that during follow-up of non-seminoma and seminoma patients the mean number of CT scans with or without contrast was 14 causing a lifetime radiation dose of 214-215 mSv and a mean annual radiation dose of $104.6 \mathrm{mSv}$. This magnitude of mean annual radiation exposure has been estimated to predispose the subjects to solid tumours at 68 times the relative risk of a control population. This estimate was based on the mode used for atomic bomb survivors (17).

Using the UNSCEAR modelling for our patients, the risk for radiation related cancer death was attributed to their diagnostic exposure but only weakly so. In case of maximal diagnostic exposure in the follow-up of eight years for each patient, it could have attributed about $2 \%$ of cancer deaths, a finding which is in concordance to that of van Walraven and collaborators (18) and well within the range given by Calandrino et al. (1). Similarly to earlier observations, a tendency to increased risk was observed among patients younger at cancer diagnosis. The younger the patient at diagnosis, the more CT-related exposure was observed in the follow-up period.

Calandrino and coworkers suggested that for testicular tumours required follow-up CT exposures (up to $23 \mathrm{CTs}$ ) determine a value for the risk of secondary fatal tumours of the same order as the fatal recurrence risk 10 to 20 years after being cured for the first cancer (1). Using different calculation models the risk estimate was $6 \%$, when the risk of recurrence estimate was $4 \%$. Thus, they evaluated absolute additional risk of second cancer induction to range between $0.1 \%$ and $10 \%$. Again the major risk determinants were age at exposure and tumour pathology.

In conclusion, patient radiation exposure in CT imaging is associated with the type of CT device and imaging protocols, which should be periodically updated and reviewed to minimize individual exposure. Using the UNSCEAR modelling $2 \%$ risk for radiation related cancer death was attributed to diagnostic exposure of study patients. Age at the diagnosis was associated with $\mathrm{CT}$ imaging related radiation exposure as the highest exposure was estimated to the youngest patients.

\section{References}

1 Calandrino, R, Ardu, V, Corletto D, del Vecchio A, Origgi D, Signorotto P, Spinelli A, Tosi G, Bolognesi A, Cariati M, Kluzer $A$ and Muscarella S: Evaluation of second cancer induction risk by $\mathrm{CT}$ follow-up in oncological long-surviving patients. Health Phys 104: 1-8, 2013.

2 https://www.cancer.gov/types/testicular/hp/testicular-treatmentpdq\#section/117

31990 Recommendations of the International Commission on Radiological Protection. ICRP publication No.60. Oxford, England: Pergamon, 1991.

4 Shrimpton PC, Jones DG, Hillier MC, Wall BF, Le Heron JC and Faulkner KL Survey of CT practice in the UK II. Dosimetric aspects. National Radiation Protection Board Publication no. R249. London, England: Her Majesty's Stationery Office (HMSO), 1991.

5 Salminen EK, Niiniviita H, Kulmala J, Määttänen H and Järvinen $\mathrm{H}$ : Radiation dose estimation in computed tomography examinations using NRPB-SR250 software in a retrospective analysis of a patient population. Rad Prot Dos 152(4) 328-33, 2012. 
6 UNSCEAR report 'Sources and effects of Ionizing radiation', volume I: sources, 2008.

7 Lee JS, Dubois SG, Coccia PF, Bleyer A, Olin RL and Goldsby RE: Increased risk of second malignant neoplasms in adolescent and young adults with cancer. Cancer 122(1): 116-123, 2016.

8 Li X, Samei E, Segars WP, Sturgeon GM, Colsher JG, Toncheva G, Yoshizumi TT and Frush DP: Patient-specific radiation dose and cancer risk estimation in CT:part II. Application to patients. Med Phys 38(1): 408-419, 2011.

9 Silva MV, Motamedinia P, Badalato GM, Hruby G and McKiernan JM: Diagnostic radiation exposure risk in a contemporary cohort of male patients with germ cell tumor. J Urol 187: 482-486, 2012.

10 Matthews JD, Forsythe A, Brady Z, Butter MW, Goergen SK, Byrnes GB, Giles GG, Wallace AB, Anderson PR, Guiver TA, McGale P, Cain TM, Gowty JG, Bickerstaffe AC and Darby SC: Cancer risk in 680000 people exposed to computed tomography scans in childhood or adolescence: data linkage study of 11 million Australians. BMJ 346: f2360, 2013.

11 Smith-Bindman R, Lipson J, Marcus R, Kim K, Mahesh M, Gould R , Berrington de A González A and Miglioretti D: Radiation dose associated with common computed tomography examinations and the associated lifetime attributable risk of cancer. Arch Intern Med 169(22): 2078-2086, 2009.

12 Pearce MS, Salotti JA, Little MP, McHugh K, Lee C, Kim KP, Howe NL, Ronckers CM, Rajaraman P, Sir Craft AW, Parker L, Berrington de and González A: Radiation exposure from CT scans in childhood and subsequent risk of leukaemia and brain tumours: a retrospective cohort study. Lancet 380(9840): 499-505, 2012.
13 Brenner DJ and Hricak H: Radiation exposure from medical imaging. Jama 304(2): 208-209, 2010.

14 Fung C, Fossa SD, Beard CJ and Travis LB: Second malignant neoplasms in testicular cancer survivors. J Natl Compr Canc Netw 10(4): 545-556, 2012.

15 Albers P, Albrecht W, Algaba F, Bokemeyer C, Cohn-Cedermark G, Fizazi K, Horwich A, Laguna MP, Nicolai N and Oldenburg J: European Association of Urology. Guidelines on Testicular Cancer: 2015 Update. Eur Urol 68(6): 1054-1068, 2015.

16 Zagars GK, Ballo MT, Lee AK and Strom SS: Mortality after cure of testicular seminoma. J Clin Oncol 22: 640, 2004.

17 Pierce DA and Preston DL: Radiation-related cancer risks at low doses among atomic bomb survivors. Radiat Res 154: 178, 2000.

18 van Walraven C, Fergusson D, Earle C, Baxter N, Alibhai S, MacDonald B, Forster AJ and Cagiannos I: Association of diagnostic radiation exposure and 2 nd abdominal pelvic malignancies after testicular cancer. JCO 29: 2883-2888, 2011.
Received October 30, 2016

Revised December 19, 2016

Accepted January 12, 2017 